\title{
Temperature-Dependent Photoluminescence Spectra of GaAsSb/AlGaAs and GaAsSbN/GaAs Single Quantum Wells under Different Excitation Intensities
}

\author{
S. A. Lourenço ${ }^{1}$, I. F. L. Dias ${ }^{1}$, J. L. Duarte ${ }^{1}$, E. Laureto ${ }^{1}$, V. M. Aquino ${ }^{1}$, and J. C. Harmand ${ }^{2}$ \\ ${ }^{1}$ Departamento de Física, Universidade Estadual de Londrina - UEL, CP6001, CEP 86051-970 Londrina, Paraná - Brazil \\ ${ }^{2}$ Laboratoire de Photonique et de Nanostructures, Centre National de la \\ Recherche Scientifique (CNRS), Route de Nozay 91460 Marcoussis, France
}

Received on 12 July, 2007

\begin{abstract}
The mechanism for low-temperature photoluminescence (PL) emissions in GaAsSb/AlGaAs and GaAsSbN/GaAs strained-layer single quantum wells (SQWs), grown by molecular-beam epitaxy, is studied in detail, using PL spectroscopy as a function of temperature and excitation intensity. In all samples, the PL peak energy as well as the full width at half maximum (FWHM), as a function of temperature, present anomalous behaviors, i.e., the PL peak energy shows a successive red/blue/redshift (S-shaped behavior) and the FWHM shows a successive blue/red/blueshift ("inverted S-shaped curve") with increasing temperature. At sufficiently low excitation intensity and in a narrow temperature interval $(50-80 \mathrm{~K})$, the nitrogen-containing samples present two clear competitive PL peaks. The low-energy PL mechanism $(8-80 \mathrm{~K})$ is dominated by localized PL transitions, while the high-energy PL mechanism is dominated by the ground state (el-hhl) PL transition. Additionally, these PL peaks show different temperature dependence with the low-energy PL peak, showing a stronger redshift than the high-energy PL peak. A competition process between localized and delocalized excitons is used to discuss these PL properties.
\end{abstract}

Keywords: Diluted III-N-V semiconductors; Photoluminescece; Quantum wells; Exciton localization

\section{INTRODUCTION}

Diluted III-N-V semiconductor alloys have recently attracted a great deal of attention due to its unusual physical properties, such as strong reduction of the band-gap energy $[1,2]$, and its possible use in GaAs-based optoeletronic and photonic devices, operating in the long-wavelength range $(1.3$ $-1.55 \mu \mathrm{m})$, which are strategic for telecommunications [3-5]. Among these materials, the $\operatorname{In}_{x} \mathrm{Ga}_{1-x} \mathrm{~N}_{y} \mathrm{As}_{1-y}$ is an attractive alloy used in active layers of lasers with pulsed and continuous wave emission at $1.31 \mu \mathrm{m}[6,7]$. However, it is difficult to obtain lasers of good quality using InGaNAs alloys at 1.55 $\mu m$ wavelength emission $[5,8]$. Recently, an alternate material based on Sb technology - GaAsSbN - grown on GaAs substrate has been used to prepare optical devices that emit light at room temperature in the $1.3-1.55 \mu \mathrm{m}$ wavelength range [9]. Particularly, $100-\AA$-thick $\mathrm{GaAs}_{0.825} \mathrm{Sb}_{0.15} \mathrm{~N}_{0.025} / \mathrm{GaAs}$ quantum wells have demonstrated emission at $1.57 \mu \mathrm{m}$ [5].

Although $\mathrm{N}$ dramatically decreases the band-gap energy of $\mathrm{N}$-based alloys, it brings a host of associated defects that make these materials to develop a strong PL property degradation [10-14]. In a previous paper, we showed that two efficient nonradiative channels are responsible for the strong thermal quenching of PL intensity in GaAsSbN/GaAs QW [12]. Many works have demonstrated that the disorder in the III-N-V alloy has a strong effect on the carrier motion, and that the radiative recombinations are generally dominated by localized excitons [10-15]. In addition, with the incorporation of nitrogen, the PL peak energy exhibits a successive red/blue/redshift with increasing temperature $[11,16]$. Although the interaction of carriers with sub-bands potential fluctuations has been used to explain the origin of the strong carrier localization observed in III-N-V QWs, this issue is still under discussion in the literature [16-18], and very few works have analyzed the

\section{GaAsSbN/GaAs system.}

In this paper, we present an analysis of the excitonic localization energy in QWs with moderate-GaAsSb/AlGaAs and high-GaAsSbN/GaAs potential fluctuation magnitude, using $\mathrm{PL}$ as a function of temperature and excitation intensity (under continuous wave excitation). The different PL spectra behavior, often observed in systems with different degrees of disorder, are observed in the GaAsSb/AlGaAs and GaAsSbN/GaAs QWs at different excitation intensities. Additionally, we have fitted the temperature dependence of the PL peak energy, using a theoretical model for the luminescence of localized states, with a Gaussian-type density of states, combined with the temperature dependence of the band gap energy. This model reproduces, quantitatively, the anomalous temperature dependence of the PL peak energy observed in our samples.

\section{EXPERIMENTAL DETAILS}

In this work, two GaAsSbN/GaAs quantum well structures with $150 \AA$ of well width $\left(\mathrm{GaAs}_{0.843} \mathrm{Sb}_{0.15} \mathrm{~N}_{0.007} / \mathrm{GaAs}\right.$ and $\mathrm{GaAs}_{0.85} \mathrm{Sb}_{0.13} \mathrm{~N}_{0.02} / \mathrm{GaAs}$ ), as well as one $\mathrm{N}$-free sample $\left(\mathrm{GaAs}_{0.8} \mathrm{Sb}_{0.2} / \mathrm{Al}_{0.3} \mathrm{Ga}_{0.7} \mathrm{As}\right)$ with $100 \AA$ of well width, were used. These samples were grown in a conventional molecularbeam epitaxy system, equipped with a radio-frequency plasma cell as the nitrogen radical source on the undoped GaAs substrate. Details on the growth conditions and the methods used to determine the alloy compositions are given in Ref. 5.

PL measures were performed using the $5145 \AA$ line of an $\mathrm{Ar}^{+}$laser focused on a $300 \mu \mathrm{m}$-diam-spot, and the temperature variation was obtained using a closed-cycle He cryostat equipped with a Lake shore temperature controller. The spectral analysis of the luminescence measurements was carried out by a grating monochromator (focal length of $0.5 \mathrm{~m}$ ), and 
detected by a thermoelectrically cooled GaInAs pin photodiode, using a standard lock-in technique.

The PL spectra shown in this paper were obtained after post-growth thermal annealing of the samples. The annealing conditions were $10 \mathrm{~min}$ at $610{ }^{\circ} \mathrm{C}$. More information on the thermal annealing can be found in Ref. 19.

\section{EXPERIMENTAL RESULTS AND DISCUSSIONS}

\section{A. Experimental results}

Figure 1 shows the PL spectra of GaAsSbN/GaAs QW with $\mathrm{N}=0.7 \%$ for different temperatures under low excitation intensity $\left(16.7 \mathrm{~W} / \mathrm{cm}^{2}\right)$. At low temperatures $(8-43 \mathrm{~K})$, the PL spectrum is dominated by only one peak, which shows a significant reduction in the intensity as the temperature increases. This thermal quenching effect was discussed in our previous work [12]. A second peak, at the higher-energy side, is observed at $\mathrm{T}>43 \mathrm{~K}$, and at $\mathrm{T}>64 \mathrm{~K}$ it starts to dominate the spectrum. The PL spectra of the low energy channel present an asymmetric behavior with a long tail of $\sim 130 \mathrm{meV}$ at the lower-energy side. This PL behavior is consistent with the tail of the DOS, characteristic of localized excitons (LE) [20-21]. However, the PL spectra of the second channel present a symmetric behavior that is a characteristic of delocalized excitons (DE).

Figure 2 shows the PL spectra for the $\mathrm{N}$-free sample (Fig. A1, A2, and A3) and for the $\mathrm{N}=0.7 \%$ sample (Fig. B1, $\mathrm{B} 2$, and B3) at a narrow temperature interval (were the competition process between the luminescence of LE and DE is more intense) and for different excitation intensities. Under low excitation intensity $\left(0.4 \mathrm{~W} / \mathrm{cm}^{2}\right)$, the PL spectra of the $\mathrm{N}$ free sample (Fig. A1) present a moderate S-shaped behavior.

However, with higher excitation intensity, this PL spectra present a similar behavior to that observed in regular III-V semiconductor materials, free of potential fluctuation [22] (see Fig. A3). On the other hand, under low excitation, the PL spectra of N-containing sample present two clear competitive peaks at temperatures between 51 and $80 \mathrm{~K}$ (Fig. B1). These peaks are labeled as L (lower energy) and $\mathrm{H}$ (higher energy). With increasing excitation intensity, the $\mathrm{H}$ peak is favored more and more so that it becomes impossible to visually separate the peaks L and $\mathrm{H}$ (Fig. B3). Then, the resulting PL peak energy $\left(E_{P L}\right)$ shows a successive red/blue/redshift displaying the so-called $\mathrm{S}$-shaped behavior.

Figure 3 shows the temperature dependence of $\mathrm{E}_{P L}$ for all samples. In the 8-33 K (Fig. 3A), 8-80 K (Fig. 3B), and 8$110 \mathrm{~K}$ (Fig. 3C) intervals, the PL peak reveals a strong dependence on the excitation intensity. With increasing excitation intensity, there is a gradual reduction in the first redshift and in the blueshift magnitudes for all samples. At sufficiently high excitation intensity, the first redshift and the blueshift disappear and the PL spectra as a function of temperature behave like in the regular III-V materials. The energy difference between the minimum position of the first redshift and the maximum position of the blueshift $\left(\Delta E_{T}\right)$ is sometimes cited in the literature as an excitation localization en-

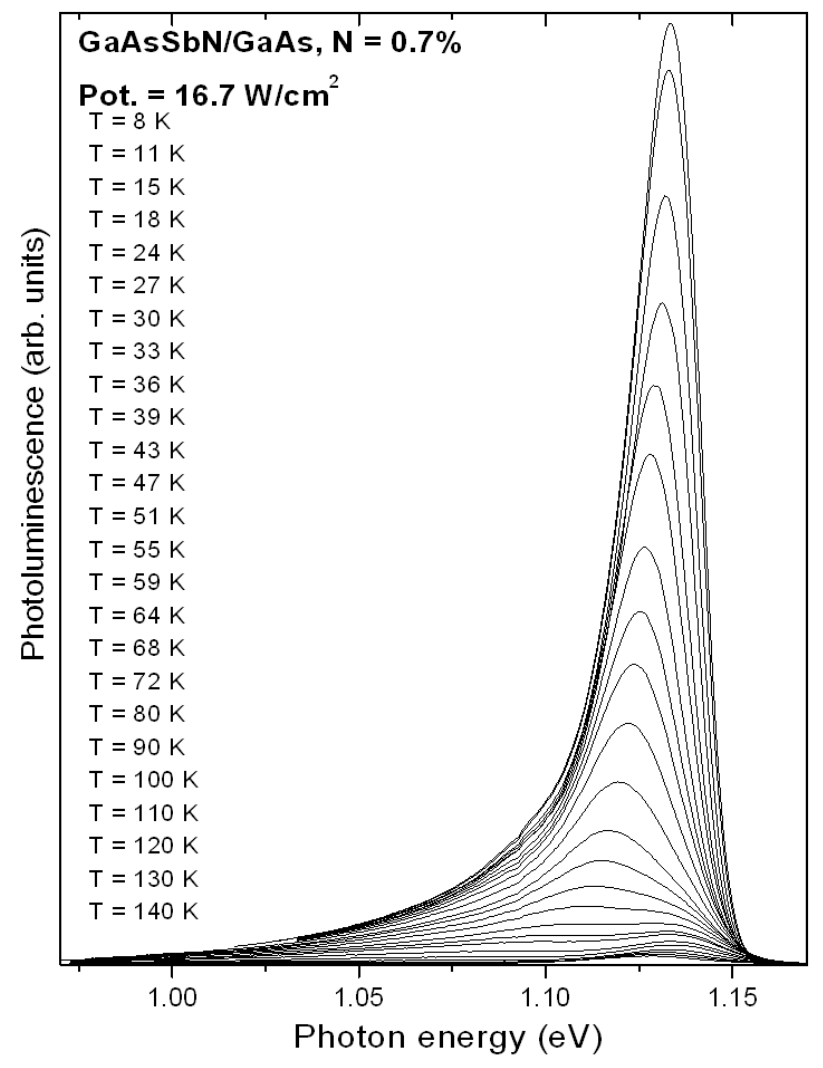

FIG. 1: PL spectra of GaAsSbN/GaAs QW with $\mathrm{N}=0.7 \%$ for different temperatures. The excitation intensity was $16.7 \mathrm{~W} / \mathrm{cm}^{2}$.

ergy [23,24]. As Fig. 3 shows, this energy difference depends strongly on the excitation intensity. At low excitation intensity (lower than $\sim 30 \mathrm{~W} / \mathrm{cm}^{2}$ for $\mathrm{N}=0.7 \%$ and $\sim 38 \mathrm{~W} / \mathrm{cm}^{2}$ for $\mathrm{N}=2.0 \%$ ) it is not possible to obtain $\Delta E_{T}$, showing that this is not a good method to estimate the localization energy in dilute nitrides (III-N-V) semiconductors. With increasing N, there is a gradual reduction of the radiative recombination efficiency. PL efficiency degradation at low $\mathrm{N}$ concentration in III-V semiconductor materials has been reported by several research groups $[10,25]$. Thus, it was not possible to obtain the PL sign for temperatures above $60 \mathrm{~K}$ with excitation intensity below $38 \mathrm{~W} / \mathrm{cm}^{2}$ in the sample with $\mathrm{N}=2.0 \%$. Fig. 3 also shows that the minimum position of the first redshift moves towards higher temperatures as the excitation increases.

Figure 4 shows the temperature dependence of the full width at half maximum (FWHM) for different excitation intensities. At T $=8 \mathrm{~K}$, the FWHM is about $9 \mathrm{meV}$ ( $\mathrm{N}$-free) and $\sim 20 \mathrm{meV}$ for $\mathrm{N}=0.7 \%$ and $\mathrm{N}=2.0 \%$. In the low temperature range, the FWHM increases rapidly with increasing temperature up to a maximum position, and then starts to decrease with the additional increase in temperature. A competition process between the $\mathrm{L}$ and $\mathrm{H}$ peaks in a definite temperature interval can explain this unusual behavior of the FWHM. At higher temperatures, the FWHM presents the regular behavior of III-V semiconductor materials, i.e., a monotonic increase with increasing temperature. The magnitude of the FWHM peak decreases with increasing excitation intensity, 

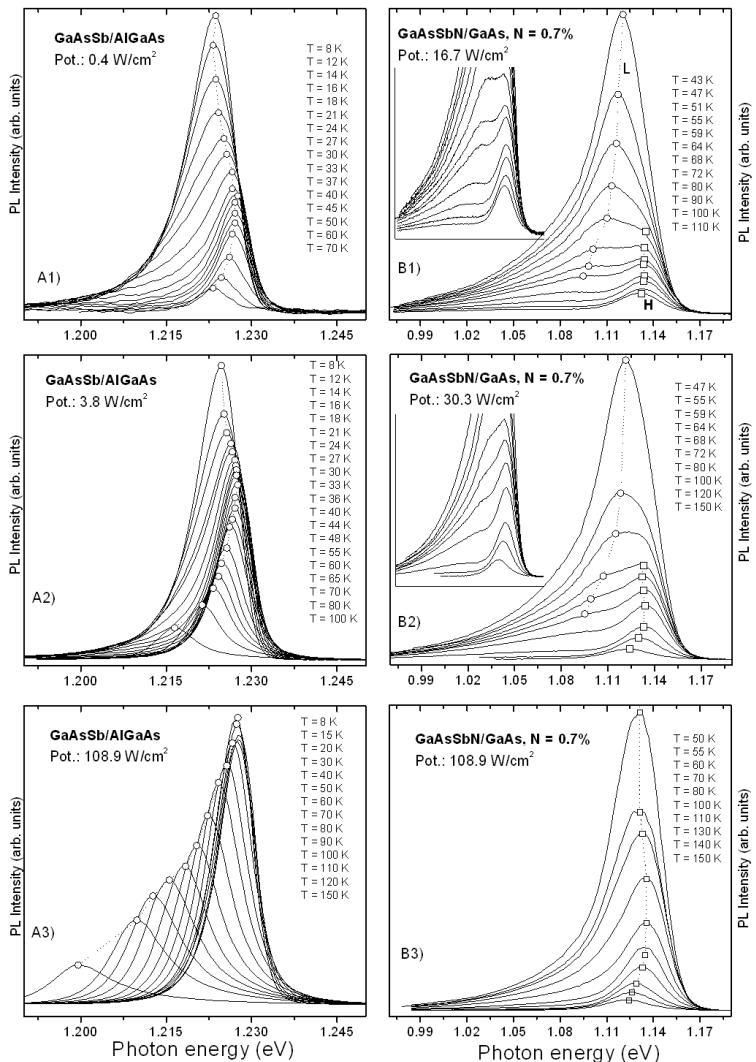

FIG. 2: PL spectra of GaAsSb/AlGaAs (A - left) and $\mathrm{GaAsSbN} / \mathrm{GaAs}$ wit $\mathrm{N}=0.7 \%$ (B - right) to a narrow temperature interval, where the emission process associated to $\mathrm{LE}$ and DE is more competitive, and for different excitation intensities. The dotted lines and empty symbols are only guides to the eyes.

and it shows, at sufficiently high excitation intensity, the regular behavior presented by semiconductor materials, free of confinement potential fluctuations.

\section{B. Discussion}

The asymmetry observed in the PL spectra of both samples, at low temperatures and low excitation intensities, with an apparent exponential tail at the low-energy side, reflects the distribution of the carriers into band-tail state of the DOS, typical of recombinations involving localized excitons $[11,20,21,26]$. According to this description, the exciton dynamic is determined by the lattice temperature, by the excitation intensity, and by the potential fluctuation magnitude (see Fig. 5). At very low temperatures, the carriers thermalize to local minima (incomplete thermalization). For increasing temperature, at low excitation intensity, the excitons have sufficient thermal energy to overcome the small potential barriers in the local potential $\left(\zeta_{1}\right)$, and they begin to thermalize and relax to the absolute potential minimum $\left(\zeta_{2}\right)$. Thus, the $\mathrm{E}_{P L}$ shifts towards a lower energy (first redshift). With an additional in-
(A)

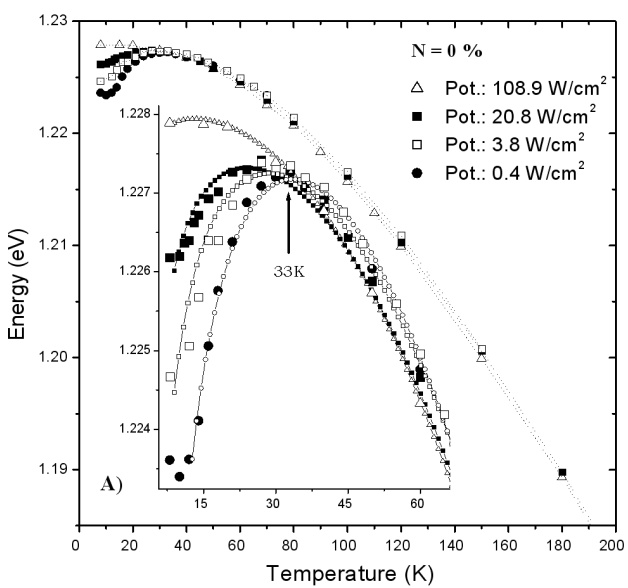

(B)

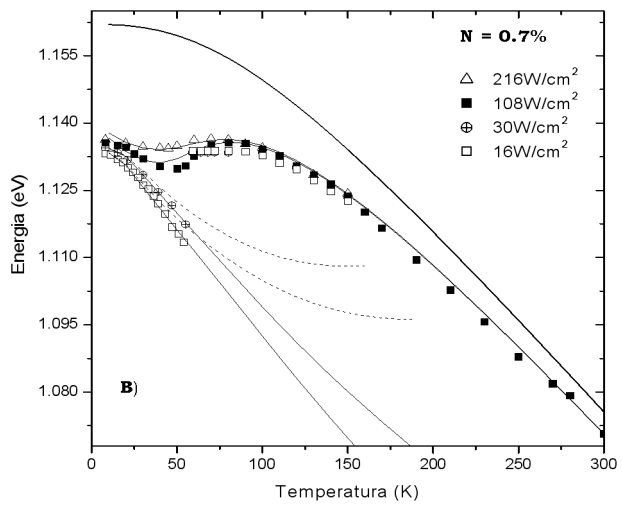

(C)

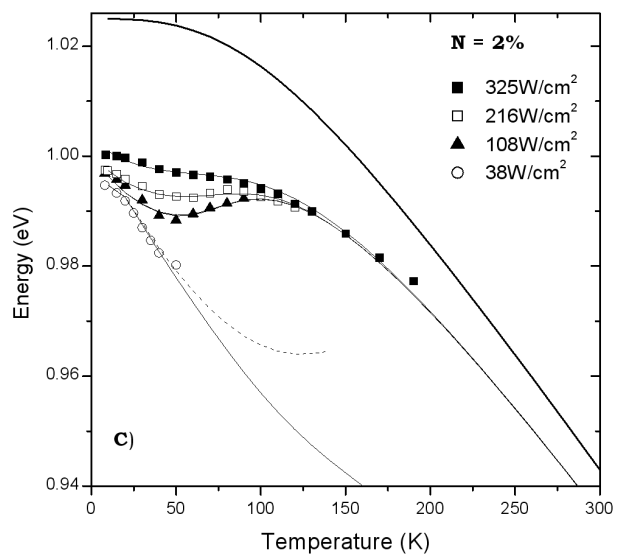

FIG. 3: PL peak energy as a function of temperature for different excitation densities. Fig. 3A), 3B) and 3C) represent the data of the samples with $\mathrm{N}$-free, $\mathrm{N}=0.7 \%$ and $\mathrm{N}=2.0 \%$, respectively. The dotted lines in 3A are only eye-guiding lines and the line-symbols curves, in the inset; represent the fittings on basis of expression (6). At low excitation intensity, the samples containing nitrogen present two peaks well separated with different temperature dependence. The solid curves in 3B and 3C represent the fittings on basis of expression (5), and the thicker solid curves represent the expression (5) without including the potential fluctuation effects, i.e., the expression (4) after having obtained the parameters with the expression (5). The dashed curves in $3 \mathrm{~B}$ and $3 \mathrm{C}$ represent the fittings on basis of expression (1), only thermal redistribution effects. 

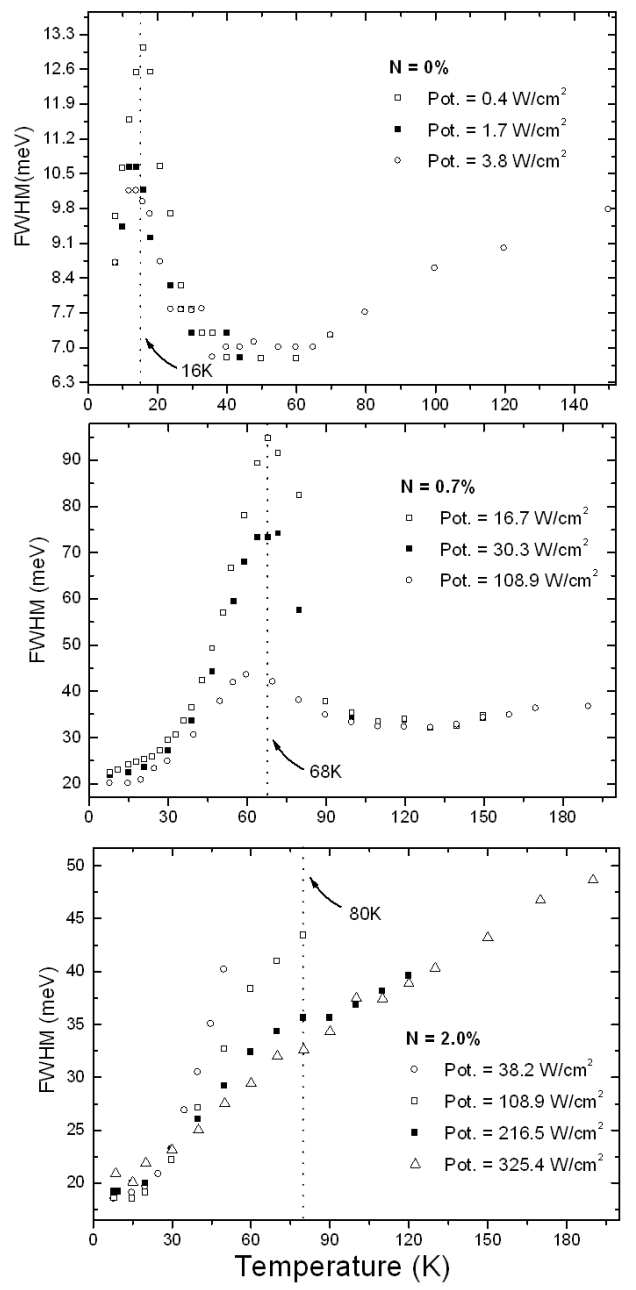

FIG. 4: FWHM as a function of temperature for the GaAsSb/AlGaAs (Fig. 4A) and GaAsSbN/GaAs (Figs. 4B and 4C) QWs.

crease in the temperature, the excitons are thermally transferred to the higher-energy states of the band-tail until reaching the conduction-band edge, shifting the $\mathrm{E}_{P L}$ toward higher energies (blueshift). With a larger increase in temperature, the thermal energy prevents the exciton localization, and the line shape of the PL spectrum becomes a characteristic line shape of the DE recombination (second redshift). Thus, the Sshaped behavior and the blue-redshift of the $\mathrm{E}_{P L}(\mathrm{~T})$, as well as the inverted S-shaped temperature dependence of the FWHM, can be qualitatively interpreted by a competition process between the recombination of localized and delocalized excitons. In this work, we show that the temperature for which this process of competition is maximum can be obtained from the positions of the FWHM peaks (Fig. 4) which are $16 \mathrm{~K}$ to Nfree, $68 \mathrm{~K}$ to $\mathrm{N}=0.7 \%$, and $\sim 80 \mathrm{~K}$ to $\mathrm{N}=2.0 \%$. Samples with stronger potential fluctuation magnitude, such as III- $\mathrm{V}_{1-x}-\mathrm{N}_{x}$, need higher thermal energy to detrap the excitons. Thus, the temperature for which the competition between the LE and
DE recombinations is maximum increases with the degree of potential disorder, as observed in our samples. In addition, as the energy of localization is greater for the $\mathrm{N}$-samples the LE and $\mathrm{DE}$ recombination peaks in the PL spectrum are enough separated so that they can be observed in a narrow temperature interval at low excitation intensities.

A difference among the shifts of the $\mathrm{E}_{P L}$ vs. T of Al$\mathrm{GaAs} / \mathrm{GaAs}$ [27,28], AlGaAs/GaAsSb and GaAs/GaAsSbN QWs with potential fluctuations, at low excitation intensity, is that the first redshift for $\mathrm{AlGaAs} / \mathrm{GaAs}$ samples is missing. In this system, the potential fluctuation is small, which means that, even at low temperature $(8 \mathrm{~K})$, the carriers get sufficient thermal energy to detrap from weakly localized states $\left(\zeta_{1}\right)$ and the thermalization is practically complete.

Figures $3 \mathrm{~B}$ and $3 \mathrm{C}$ show that the $\mathrm{L}$ peak presents a stronger temperature-induced redshift than the $\mathrm{H}$ peak. This effect can be explained by the different origin of the temperature dependence of $\mathrm{L}$ and $\mathrm{H}$ peaks. The first redshift ( $\mathrm{L}$ peak) arises from a carrier-redistribution process, i.e., the transfer of thermally activated excitons from a higher- to a lower-energy band-tail state. The second redshift ( $\mathrm{H}$ peak) is due to the band-gap temperature dependence, i.e., a cumulative effect of thermal lattice expansion and electron-phonon interaction [22]. Recently, T. Khee Ng et al.[16] detected a similar anomalous temperature-dependent photoluminescence characteristic of as-grown GaInNAs/GaAs QW, with two-segmented trend in the PL peak energy vs. temperature. They fitted the twosegmented temperature dependence of the PL peak energy using the Varshni expression [29]. In this study, we show that the anomalous temperature-dependent photoluminescence characteristic is also observed in post-growth thermal annealing GaAsSbN/GaAs QWs, at low excitation intensity, and that the lower energy PL peak can be fitted using a thermal carrier redistribution model.

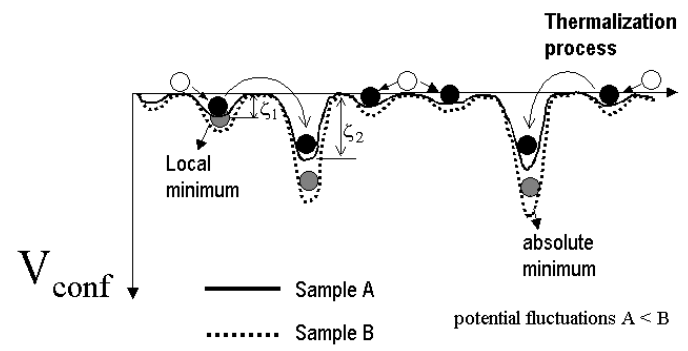

FIG. 5: Schematic representation of confinement potential, $\mathrm{V}_{\text {conf }}$, for two samples with different confinement potential fluctuations, sample A and sample B. Empty circles represent the delocalized excitons. The arrows indicate the relaxation process to the local or absolute potential minima. Filled circles represent the localized excitons at these minima. Dotted line represents the profile of confinement potential to the sample with bigger magnitude of the potential fluctuation (sample B). $\zeta_{1}$ and $\zeta_{2}$ represent the potential barrier heights for wells of the local minima and of the absolute minima, respectively.

As the density of the band-tail states is finite, with the increase of the excitation intensity there is a gradual filling of the states with lower energy in the band-tail. This effect is re- 
sponsible for the decrease of the blueshift observed in Fig. 3. For sufficiently high-excitation intensities, the transitions related to the band-tail states saturate, and the recombination of the DE becomes more efficient. Thus, the relative intensity of the DE recombination continues to increase with increasing excitation density, and the blueshift of the PL peak saturates. These trends (behavior of PL peak with excitation intensity) are confirmed by our samples as well as in GaAsN/GaAs [30] and InGaAsN/GaAs [24] QWs. The blueshift of the PL peak energy, caused by an increase in the excitation intensity, as well as saturation behavior at higher levels of excitation intensity, has been observed in alloys containing dot-like localized states [31].

The nature of the disorders in III- $\mathrm{V}_{1-x}-\mathrm{N}_{x}$ semiconductors materials that lead to a band-tail states in the DOS can be interpreted as an inhomogeneous distribution of nitrogen atoms in alloy. In highly diluted III-V-N alloys $(\mathrm{N}<0.2 \%)$, the $\mathrm{N}$ insertion gives rise to discrete eletronic levels related to isolated single $\mathrm{N}$ atoms and to $\mathrm{N}-\mathrm{N}$ pairs or clusters [32-37]. Within such limit, the low temperature PL spectra are characterized by a number of sharp lines (line width $\sim 0.5 \mathrm{meV}$ ) due to the recombination of excitons localized in $\mathrm{N}$ complexes [37]. The strongly localized character of the $\mathrm{N}$ isoeletronic traps leads to a strong local potential fluctuation for electrons and holes, resulting therefore in a semiconductor quantum dot behavior [33,36]. Indeed, fluctuations of $\mathrm{N}$ compositions at $1 \%$ only leads to an $\sim 180 \mathrm{meV}$ change in the band-gap energy of $\mathrm{GaAs}_{1-x} \mathrm{~N}_{x}$ [38-40]. The localization of excitons on quantumdot-like composition fluctuations has been directly observed in temperature-dependent near-field photoluminescence spectra of InGaAsN [41] and GaNAs [42]. For increasing N concentration, the PL from pairs states becomes an asymmetric line shape with a sharp high-energy cut-off and a long lowenergy tail $[25,36,37,43]$. In a quaternary material such as GaInNAs and GaAsSbN, the situation is even more complex. The presence of a fourth element- In or Sb- at the proximity of $\mathrm{N}$ atoms can significantly alter the energy of $\mathrm{N}$-related localized states. Therefore, the QW structures based on III$\mathrm{V}_{1-x}-\mathrm{N}_{x}$ present a very high potential fluctuation resulting in a complex band tailing.

\section{Theoretical approach}

As shown in previous discussions, the emission spectra, in our materials, are composed of a radiative recombination of localized and delocalized states. Considering the radiative recombination, the thermal escape, and the re-capture of the excitons in a localized state ensemble Li et al. $[45,46]$ derive a distribution function of localized carries from a rate equation under quasi-steady state. Assuming that the localized state has a Gaussian-type energetic distribution of density of states, the luminescence spectrum of the localized excitons can be found. According to this model, the temperature dependence of the PL peak position is given by:

$$
E(T)=E_{0}-x(T) K_{B} T
$$

The second term represents the effect of thermal redistribution of localized carriers, and $k_{B}$ is the Boltzmann constant. The dimensionless coefficient $x(\mathrm{~T})$ can be obtained by solving numerically the following equation:

$$
x e^{x}=\left[\left(\frac{\sigma}{K_{B} T}\right)^{2}-x\right]\left(\frac{\tau_{r}}{\tau_{t r}}\right) e^{\left(E_{0}-E_{a}\right) / K_{B} T}
$$

where $E_{0}$ and $\sigma$ are the central energy and broadening parameter for the distribution of the localized states, respectively. $\tau_{t r}$ is the carrier transfer time and $\tau_{r}$ is the carrier recombination time. Like the Fermi level in the Fermi-Dirac distribution, $E_{a}$ gives a special energy level below which the localized states are occupied by the excitons at $0 \mathrm{~K}$. It shows that the magnitude and sign of $\left(E_{a}-E_{0}\right)$ affects strongly the temperature dependence of the PL peak $[45,46]$.

In a high-temperature region, where $\left(\tau_{r} / \tau_{t r}\right) \exp \left[\left(E_{0}-\right.\right.$ $\left.\left.E_{a}\right) / k_{B} T\right]>>1$, the approximated solution of Eq. (2) is $\left(\sigma / K_{B} T\right)^{2}$, and the Eq.(1) becomes:

$$
E(T) \approx E_{0}-\frac{\sigma^{2}}{k_{B} T}
$$

which is the band-tail model proposed by Eliseev et al. [47].

On the other hand, the temperature dependence of the band gap energy in semiconductor materials can be described through the following relation proposed by Pässler [48]:

$$
E_{g}(T)=E_{g}(T=0)-\frac{\alpha \Theta}{2}\left[\sqrt[p]{1+\left(\frac{2 T}{\Theta}\right)^{p}}-1\right]
$$

where $E_{g}(T=0)$ is the energy gap at zero Kelvin temperature, $\alpha \equiv S(\infty) \equiv-(d E(T) / d T)_{T \rightarrow \infty}$ is the high-temperature limit value for the forbidden gap entropy, $\Theta$ is a characteristic temperature parameter of the material representing the effective phonon energy $\hbar w=k_{B} \Theta$ in units of absolute temperature, and $p$ is an empirical parameter related to the shape of the electron-phonon spectral functions [48]. This expression shows better fitting for the temperature dependence of the band-gap energy for several semiconductor materials, particularly at the low temperature region, where the Varshni [29] model did not show good results.

Introducing the expressions (1) or (3) in equation (4), it is possible to obtain functions that fit the temperature dependence of the PL peak energy, in situations where the potential fluctuations are relevant. These relations are given by:

$$
E_{P L}(T)=\left\{E_{0}-\frac{\alpha \Theta}{2}\left[\sqrt[p]{1+\left(\frac{2 T}{\Theta}\right)^{p}}-1\right]\right\}-x(T) k_{B} T
$$

$$
E_{P L}(T)=\left\{E_{0}-\frac{\alpha \Theta}{2}\left[\sqrt[p]{1+\left(\frac{2 T}{\Theta}\right)^{p}}-1\right]\right\}-\frac{\sigma_{E}^{2}}{K_{B} T}
$$


TABLE I: The parameters after fitting the experimental data on basis of Equations (5) and (6).

\begin{tabular}{|c|c|c|c|c|c|c|c|c|}
\hline & Excit. $\left(\mathrm{W} / \mathrm{cm}^{2}\right)$ & \begin{tabular}{|l}
$\mathrm{E}_{0}$ \\
$(\mathrm{eV})$
\end{tabular} & $\begin{array}{l}\alpha \\
(\mathrm{meV} / \mathrm{K})\end{array}$ & $\begin{array}{l}\Theta \\
(\mathrm{K})\end{array}$ & $\mathrm{p}$ & $\begin{array}{l}\sigma \\
(\mathrm{meV})\end{array}$ & $\tau_{r /} \tau_{t r}$ & $\begin{array}{l}\mathrm{E}_{0}-\mathrm{E}_{a} \\
(\mathrm{meV})\end{array}$ \\
\hline \multirow{4}{*}{$\mathrm{N}=0$} & 108.9 & 1.2282 & 0.43 & 216.2 & 2.54 & 0.5 & - & - \\
\hline & 20.8 & 1.2287 & 0.43 & 216.2 & 2.54 & 1.43 & - & - \\
\hline & 3.8 & 1.2294 & 0.43 & 216.2 & 2.54 & 1.94 & - & - \\
\hline & 0.4 & 1.2311 & 0.43 & 189.8 & 2.54 & 2.83 & - & - \\
\hline \multirow{4}{*}{$\mathrm{N}=0.7$} & 216 & 1.1622 & 0.431 & 216.2 & 2.54 & 11.47 & 13 & 20.5 \\
\hline & 108 & 1.1620 & 0.431 & 216.2 & 2.54 & 11.47 & 47 & 20.5 \\
\hline & 30 & 1.1344 & 0.431 & 216.2 & 2.54 & 22.44 & 47 & -13.9 \\
\hline & 16 & 1.1334 & 0.431 & 216.2 & 2.54 & 29.74 & 47 & -12.9 \\
\hline \multirow{4}{*}{$\mathrm{N}=2.0$} & 325 & 1.025 & 0.441 & 240.89 & 2.99 & 15.81 & 3 & 21.2 \\
\hline & 216 & 1.025 & 0.441 & 240.89 & 2.99 & 15.11 & 7 & 24.2 \\
\hline & 108 & 1.025 & 0.441 & 240.89 & 2.99 & 14.71 & 25 & 24.2 \\
\hline & 38 & 1.025 & 0.441 & 240.89 & 2.99 & 20.71 & 247 & -15.5 \\
\hline
\end{tabular}

Based on expression (6), the best numerical fit for our experimental data ( $\mathrm{GaAsSb} / \mathrm{AlGaAs})$ is represented by the linesymbol curves in the inset of Fig. (3A), and the best numerical fit, based on expression (5), is represented by the solid curves in the Figs. (3B) and (3C) for GaAsSbN/GaAs samples with $\mathrm{N}$ $=0.7 \%$ and $\mathrm{N}=2.0 \%$, respectively. The parameters obtained after fitting the experimental data are shown in Table 1.

When $E_{0}-E_{a}$ takes a positive value, the S-shaped temperature behavior of the PL peak energy can be well reproduced, as shown in figures $3 \mathrm{~B}$ and $3 \mathrm{C}$. Similar result was obtained for the InGaAsN material [46]. In low excitation regions, where it is possible to follow separately the energy variation of the $\mathrm{L}$ peak with the temperature $\left(E_{P L}^{L}(T)\right.$ and the energy variation of the $H$ peak $\left(E_{P L}^{H}(T)\right)$, the dependence of $E_{P L}^{L}(T)$ was obtained taking into consideration the negative values for $E_{0}-E_{a}$. The negative value for $E_{0}-E_{a}$ is in agreement with the results obtained by Li et al.[45] for InGaN alloy with In-rich clusters and by $\mathrm{Xu}$ et al. [49] for self-organized InAs quantum dots. When $E_{0}-E_{a}$ is negative, the PL peak energy decreases rapidly as the temperature increases. This effect is produced by the thermal redistribution of the carriers in localized states.

The inset of Fig. 3A shows that the $\mathrm{E}_{P L}$, obtained from a sample with $\mathrm{N}=0$, behaves differently with the excitation intensity, depending on the temperature of the sample. For $\mathrm{T}<33 \mathrm{~K}$, the $\mathrm{E}_{P L}$ increases with increasing the excitation intensity, whereas for $\mathrm{T}=33 \mathrm{~K}$ the $\mathrm{E}_{P L}$ remains practically constant. For $\mathrm{T}>33 \mathrm{~K}$, the $\mathrm{E}_{P L}$ decreases with increasing the excitation intensity. This $\mathrm{E}_{P L}$ versus excitation intensity behavior can be understood as a competition process between the band-gap energy renormalization (at sufficiently high carrier densities the band-gap energy decreases with increasing excitation intensity [50]) and the blueshift of $\mathrm{E}_{P L}$ due to potential fluctuations. The fittings of the $\mathrm{E}_{P L}(\mathrm{~T})$, for different excitation intensities, were obtained by varying only the $\mathrm{E}_{0}$ and $\sigma$ parameters in expression (6). $\alpha, \Theta$ and $p$ values were considered constant. Such fittings are represented in the inset of Fig. 3A by line-symbols curves, and the parameters obtained are listed in Table 1. The decrease in excitation intensity is followed by the consistent increase in $\sigma$ and $\mathrm{E}_{0}$ values. $\sigma$ can be analyzed as the degree of screening of the localized states by the carriers density. If $\sigma$ is null (high excitation intensity), the number of carriers are so high that the fluctuations are screened. In this case, $\mathrm{E}_{P L}(\mathrm{~T})$ must follow the temperature dependence of the band-gap energy. If $\sigma$ increases (lower excitation intensity) the carrier-redistribution process, in the localized states, dominates the variation of the $\mathrm{E}_{P L}(\mathrm{~T})$ at low temperature interval. As excitation intensity was reduced, the $\mathrm{E}_{0}$ increases due to lower band-gap renormalization and the increase of the blueshift range of the $\mathrm{E}_{P L}$ curve due to lowering of the band tail filling. These different process (carriers thermal redistribution and the band-gap renormalization effect) generate a crossing of the $\mathrm{E}_{P L}(\mathrm{~T})$ curves obtained in different excitation intensities. In the case of $\mathrm{N}$-free samples, this crossing occurred at $\mathrm{T} \approx 33 \mathrm{~K}$. We have observed a similar temperature dependence of the $\mathrm{E}_{P L}(T)$ for different excitation intensities in $\mathrm{AlGaAs} / \mathrm{GaAs}$ single QWs [52]. In this case, the crossing temperature occurred at $\approx 30 \mathrm{~K}$. More details about this discussion can be seen in a future publication [53].

\section{CONCLUSIONS}

In conclusion, the recombination process associated with the PL emission in post-growth thermal annealing GaAsSbN/GaAs and GaAsSb/AlGaAs quantum wells was discussed. Through the analysis of the PL spectra as a function of temperature combined with the variation of excitation intensity, the S-shaped emission behavior, the blueshift and the FWHM peak, often observed in the temperaturedependent PL spectra from disordered systems [16-18], were observed in our systems and explained by a competition process between the localized excitons to band-tail states in the DOS and the delocalized excitons related to the recombination of the $n=1$ electrons in QW with holes. We showed that the temperature for which this competition process is maximum can be obtained from the positions of the FWHM peaks. The band-tail states in our N-free and Ncontaining samples may originate principally from the com- 
position nonuniformity of the $\mathrm{Sb} / \mathrm{Al}$ and the $\mathrm{N}$, respectively. Since similar results were found for GaNAs/GaAs [18] and GaInNAs/GaAs [17] we suggest that the strong local fluctuations in the $\mathrm{N}$ concentration have the greatest effect on the recombination dynamics in GaAsSbN quaternary alloys.

The S-shaped emission behavior observed in our systems containing $\mathrm{N}$ was also observed in thin QWs systems of InGaP/GaAs ( $L_{w}=15$ and $30 \AA$ ) [44], showing that the anomalous PL-temperature dependence reported in this paper is not exclusive of samples containing $\mathrm{N}$, but of systems with high potential fluctuation magnitude.

In addition, this study used a combination between a thermal distribution model for carriers in localized states of DOS $[45,46]$ with the model proposed by Pässler [48] for the temperature dependence of the band-gap energy to fitting the temperature dependence of the PL peak energy. This compound- model provided an adequate description of the S-shaped emission behavior observed in our samples containing $\mathrm{N}$ for sufficiently high excitation intensities and the intense redshift of the PL peak energy with T for low excitation intensities.

\section{Acknowledgments}

The authors would like to acknowledge the financial support granted by the Brazilian agencies: Coordenação de Aperfeiçoamento de Pessoal de Nível Superior (CAPES), Conselho Nacional de Desenvolvimento Científico e Tecnológico $(\mathrm{CNPq})$, Fundação Araucária de Apoio ao Desenvolvimento Científico e Tecnológico do Paraná (Fundação Araucária), and Fundação Banco do Brasil (FBB).
[1] H. Saito, T. Makimoto, and N. Kobayashi, J. Cryst. Growth, 195,416 (1998)

[2] W. Shan, W. Walukiewicz, J. W. Ager III, E. E. Haller, J. F. Geisz, D. J. Friedman, J. M. Olson, and Sarah R. Kurtz, J. Appl. Phys. 86, 2349 (1999).

[3] M. Kondow, T. Kitatani, S. Nakatsuka, M. C. Larson, K. Nakahara, Y. Yazawa, M. Okay, and K. Uomi, IEEE, J. Setect. Topics in Q. Electron. 3, 719 (1997).

[4] J. F. Geisz and D. J. Friedman, Semicond. Sci. Technol. 17, 769 (2002).

[5] J. C. Harmand, G. Ungaro, J. Ramos, E. V. K. Rao, G. SaintGirons, R. Taissier, G. Lê Roux, L. Largeau, and G. Patriarche, J. Cryst. Growth 227, 553 (2001).

[6] K. Nakahara, M. Kondow, T. Kitatani, M. C. Larson, and K. Uomi, IEEE. Photon. Technol. Lett. 10, 487 (1998).

[7] S. Sato, Y. Osawa, T. Saitoh, and I. Fujimura, Electronics. Lett. 33 (1997) 1386.

[8] V. M. Ustinov and A. E. Zhukov, Semicond. Sci. Technol. 15, R41(2000).

[9] G. Ungaro, G. Le Roux, R. Teissier and J. C. Harmand, Eletronics Lett. 35, 1246 (1999).

[10] I. A. Buyanova, W. M. Chen, G. Pozina, J. P. Bergman, B. Monemar, H. P. Xin, and C. W. Tu, Appl. Phys. Lett. 75, 501 (1999).

[11] A. Polimeni, M. Capizzi, M. Geddo, M. Fischer, M. Reinhardt, and A. Forchel, Appl. Phys. Lett. 77, 2870 (2000).

[12] S. A. Lourenço, I. F. L. Dias, L. C. Poças, J. L. Duarte, J. B. B. de Oliveira, and J. C. Harmand, J. Appl. Phys. 93, 4475 (2003)

[13] L. Grenouillet, C. Bru-Chevallier, G. Guillot, P. Gilet, P. Duvaut, C. Vannuffel, A. Million, and A. Chenevas-Paule, Appl. Phys. Lett. 76, 2241 (2000).

[14] H. P. Xin, K. L. Kavanagh, Z. Q. Zhu, and C. W. Tu, Appl. Phys. Lett. 74, 2337 (1999).

[15] M. A. Pinault and E. Tounie, Appl. Phys. Lett. 78, 1562 (2001).

[16] T. K. Ng, S. F. Yoon, W. K. Loke, S. Wicaksono, J. Cryst. Growth 270, 351 (2004).

[17] S. Shirakata, M. Kondow, and T. Kitatani, Appl. Phys. Lett. 80, 2087 (2002).

[18] X. D. Luo, Z. Y. Xu, Z. Pan, L. H. Li, and Y. W. Lin, Appl. Phys. Lett. 79, 958 (2001).

[19] J. C. Harmand, A. Caliman, E. V. K. Rao, L. Largeau, J. Ramos, R Teissier, L. Travers, G. Ungaro, B. Theys, and I. F. L. Dias, Semicond. Sci. Thechnol. 17, 77 8(2002).
[20] Djamel Ouadjaout, Yves Marfaing, Phys. Rev. B 46, 7908 (1992).

[21] A. Aït-Ouali, J. L. Brebner, R. Y. -F. Yip, and R. A. Masut, J. Appl. Phys. 86, 6803 (1999), and references therein.

[22] S. A. Lourenço, I. F. L. Dias, J. L. Duarte, E. Laureto, D. O. Toginho Filho, E. A. Meneses, and J. R. Leite, Eur. Phys. J. B 21, 11 (2001), and references therein.

[23] B. Q. Sun, D. S. Jiang, Z. Pan, L. H. Li, and R. H. Wu, Appl. Phys. Lett. 77, 4148 (2000).

[24] S. Shirakata, M. Kondow, and T. Kitatani, Appl. Phys. Lett. 79, 54 (2001).

[25] I. A. Buyanova,W. M. Chen, B. Monemar, H. P. Xin, and C.W. Tu, Mater. Sci. Eng. B 75, 166 (2000).

[26] M. Grassi, A. F. Fragano, A. Patané, M. -Capazi, E. Runge, and R. Zimmermann, Phys. Rev. B 61, 10985 (2000), and references therein.

[27] C. Delalande, M. H. Meynadier, and M. Voos, Phys. Rev. B 31, 2497 (1984).

[28] M. Gurioli, A. Vinattieri, J. Martinez-Pastor, and M. Colocci, Phys. Rev. B 50, 11817 (1994).

[29] Y. P. Varshni, Physica 34, 194 (1967).

[30] F. Saidi, F. Hassen, H. Maaref, L. Auvray, H. Dumont, Y. Monteil, Mater. Sci. Eng. C 21, 245 (2002).

[31] T. Mattila, S. Wei, and A. Zunger, Phys. Rev. Lett. 83, 2010 (1999).

[32] X. Liu, M. -E. Pistol, and L. Samuelson, Phys. Rev. B 42, 7504 (1990).

[33] P. Leroux-Hugon and H. Mariette, Phys. Rev. B 30, 1622 (1984).

[34] S. Francouer, S. A. Nikishin, C. Jin, Y. Qiu, and H. Temkin, Appl. Phys. Lett. 75, 1538 (1999).

[35] T. Makimoto, H. Saito, T. Nishida, and N. Kobayashi, Appl. Phys. Lett. 70, 2984 (1997).

[36] P. C. Kent and A. Zunger, Phys. Rev Lett. 86, 2613 (2001).

[37] Y. Zhang, B. Fluegel, A. Mascarenhas, H. P. Xin, and C. W. Tu, Phys. Rev. B 62, 4493 (2000); 63, 085205 (2001).

[38] S. -H. Wei, and A Zunger, Phys. Rev. Lett. 76, 664 (1996).

[39] W. Shan, W. Walukiewicz, J. W. Ager, E. E. Haller, J. F. Geisz, D. J. Friedman, J. M. Olson, and S. R. Kurtz, Phys. Rev. Lett. 82, 1221 (1999).

[40] J. D. Perkins, A. Mascarenhas, Y. Zhang, J. F. Geisz, D. J. Friedman, J. M. Olson, and S. R. Kurtz, Phys. Rev. Lett. 82, 
3312 (1999).

[41] A. M. Mintairov, T. H. Kosel, J. L. Merz, P. A. Blagnov, A. S. Vlasov, V. M. Ustinov, and R. E. Cook, Phys. Rev. Lett. 87, 277401 (2001)

[42] K. Matsuda, T. Saiki, M. Takahashi, A. Moto, and S. Takagishi, Appl. Phys. Lett. 78, 1508 (2001).

[43] H. P. Xin and C. W. Tu, Appl. Phys. Lett. 76, 1267 (2000); 77, $2180(2000)$.

[44] E. Laureto, E. A. Meneses, W. Carvalho Jr, A. A. Bernussi, E. Ribeiro, E. C. F. da Silva, and J. B. B. de Oliveira, Braz. J. Phys. 32, 314 (2002); complementary measurements of PL as a function of temperature and excitation intensity were obtained by us for the InGaP/GaAs system. These complementary measurements are not shown here.

[45] Q. Li, S. J. Xu, W. C. Cheng, M. H. Xie, and S. Y. Tong, Appl. Phys. Lett. 79, 1810 (2001).
[46] Q. Li, S. J. Xu, M. H. Xie, and S. Y. Tong, J. Phys.: Condens. Matter 17, 4853 (2005).

[47] P. G. Eliseev, J. A. Phys. 93, 5404 (2003).

[48] R. Pässler, Phys. Status. Solidi. 200, 155 (1997).

[49] Z. Y. Xu, Z. D. Lu, Z. L. Yuan, X. P. Yang, B. Z. Zheng, J. Z. $\mathrm{Xu}$, W. K. Ge, Y. Wang, J. Wang, L. L. Chang, Superlattice and Microstruct, 23, 381, (1998).

[50] R. Cingolani and K. Ploog, Adv. Phys. 40, 535 (1991).

[51] N. A. Poklonski and A. I. Siaglo, Phys. Solid. State, 43, 157 (2001).

[52] S. A. Lourenço, M. A. T. da Silva, I. F. L. Dias, J. L. Duarte, E. Laureto, A. A. Quivy, and T. E. Lamas, J. Appl. Phys. 101, 113536 (2007).

[53] J. L. Duarte, L. C. Poças, E. Laureto, I. F. L. Dias, E. M. Lopes, S. A. Lourenço, and J. C. Harmand (to be submitted). 